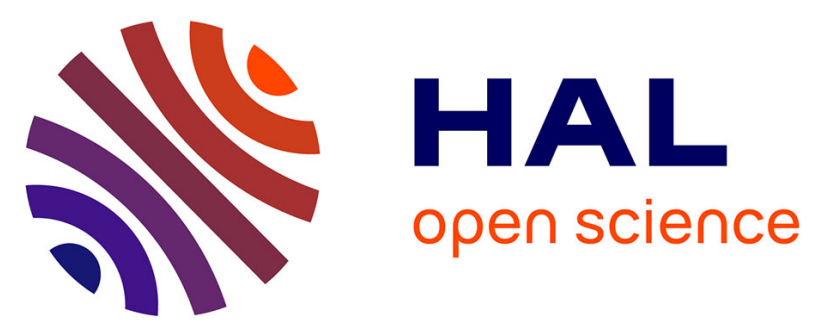

\title{
Automated Quantification of Right Ventricular Fat at Contrast-enhanced Cardiac Multidetector CT in Arrhythmogenic Right Ventricular Cardiomyopathy
}

Hubert Cochet, Arnaud Denis, Yuki Komatsu, Amir S. Jadidi, Tassadit Aït Ali, Frédéric Sacher, Nicolas Derval, Jatin Relan, Maxime Sermesant, Olivier Corneloup, et al.

\section{To cite this version:}

Hubert Cochet, Arnaud Denis, Yuki Komatsu, Amir S. Jadidi, Tassadit Ait Ali, et al.. Automated Quantification of Right Ventricular Fat at Contrast-enhanced Cardiac Multidetector CT in Arrhythmogenic Right Ventricular Cardiomyopathy. Radiology, 2015, 275 (3), pp.683-91. 10.1148/radiol.14141140 . hal-01244219

\author{
HAL Id: hal-01244219 \\ https://hal.inria.fr/hal-01244219
}

Submitted on 15 Dec 2015

HAL is a multi-disciplinary open access archive for the deposit and dissemination of scientific research documents, whether they are published or not. The documents may come from teaching and research institutions in France or abroad, or from public or private research centers.
L'archive ouverte pluridisciplinaire HAL, est destinée au dépôt et à la diffusion de documents scientifiques de niveau recherche, publiés ou non, émanant des établissements d'enseignement et de recherche français ou étrangers, des laboratoires publics ou privés. 
Automated Quantification of Right Ventricular Fat at Contrastenhanced Cardiac Multidetector CT in Arrhythmogenic Right Ventricular Cardiomyopathy ${ }^{1}$

Hubert Cochet, MD, PhD

Arnaud Denis, MD

Yuki Komatsu, MD

Amir S. Jadidi, MD

Tassadit Aït Ali, MD

Frédéric Sacher, MD, PhD

Nicolas Derval, MD

Jatin Relan, PhD

Maxime Sermesant, PhD

Olivier Corneloup, MD

Mélèze Hocini, MD

Michel Haïssaguerre, MD

François Laurent, MD

Michel Montaudon, MD, PhD

Pierre Jaïs, MD
${ }^{1}$ From the Department of Cardiovascular Imaging (H.C., T.A.A., O.C., F.L., M.M.) and Department of Cardiac Pacing and Electrophysiology (A.D., Y.K., F.S., N.D., M. Hocini, M. Haïssaguerre, P.J.), Hôpital Cardiologique Haut Lévêque, CHU/Université de Bordeaux, Avenue Magellan, 33604 Pessac, France; L'Institut de Rythmologie et de Modélisation Cardiaque LIRYC, CHU/Université de BordeauX/INSERM U1045, Pessac, France (H.C., A.D., Y.K., F.S., N.D., M. Hocini, M. Haïssaguerre, F.L., M.M., P.J.); Arrhythmia Department, University Heart Center, Freiburg-Bad Krozingen, Germany (A.S.J.); and Inria Asclepios Research Team, Sophia Antipolis, France (J.R., M.S.). Received June 2, 2014; revision requested July 2; revision received September 8; accepted October 10; final version accepted November 6. Supported by Fondation Leducq (grant 09 CVD 03) and Agence Nationale de la Recherche (grants ANR-10-IAHU-04 and ANR-11-EQPX-0030). Address correspondence to H.C. (e-mail: hcochet@wanadoo.fr).
Purpose:

Materials and Methods:

Results:

Conclusion:
To evaluate an automated method for the quantification of fat in the right ventricular (RV) free wall on multidetector computed tomography (CT) images and assess its diagnostic value in arrhythmogenic RV cardiomyopathy (ARVC).

This study was approved by the institutional review board, and all patients gave informed consent. Thirty-six patients with ARVC (mean age \pm standard deviation, 46 years \pm 15; seven women) were compared with 36 age- and sexmatched subjects with no structural heart disease (control group), as well as 36 patients with ischemic cardiomyopathy (ischemic group). Patients underwent contrast materialenhanced electrocardiography-gated cardiac multidetector CT. A 2-mm-thick RV free wall layer was automatically segmented and myocardial fat, expressed as percentage of RV free wall, was quantified as pixels with attenuation less than -10 HU. Patient-specific segmentations were registered to a template to study fat distribution. Receiver operating characteristic (ROC) analysis was performed to assess the diagnostic value of fat quantification by using task force criteria as a reference.

Fat extent was $16.5 \% \pm 6.1$ in ARVC and $4.6 \% \pm 2.7$ in non-ARVC $(P<.0001)$. No significant difference was observed between control and ischemic groups $(P=.23)$. A fat extent threshold of $8.5 \%$ of RV free wall was used to diagnose ARVC with $94 \%$ sensitivity (95\% confidence interval $[\mathrm{CI}]: 82 \%, 98 \%$ ) and $92 \%$ specificity (95\% CI: $83 \%, 96 \%)$. This diagnostic performance was higher than the one for RV volume (mean area under the ROC curve, $0.96 \pm 0.02$ vs $0.88 \pm 0.04 ; P=.009)$. In patients with ARVC, fat correlated to RV volume $(R=0.63, P<.0001)$, RV function $(R=-0.67, P=.001)$, epsilon waves $(R=$ $0.39, P=.02)$, inverted $\mathrm{T}$ waves in $\mathrm{V}_{1}-\mathrm{V}_{3}(R=0.38, P=$ $.02)$, and presence of $P K P 2$ mutations $(R=0.59, P=.02)$. Fat distribution differed between patients with ARVC and those without, with posterolateral RV wall being the most ARVC-specific area.

Automated quantification of RV myocardial fat on multidetector CT images is feasible and performs better than RV volume in the diagnosis of ARVC.

${ }^{\circ}$ RSNA, 2015 
A rrhythmogenic right ventricular cardiomyopathy (ARVC) is a major cause of ventricular arrhythmia, congestive heart failure, and sudden death (1). The diagnosis of ARVC is complex and requires compilation of clinical, electrophysiologic, structural, and mechanical features (2). The disease is characterized by fibro-fatty replacement of normal myocardial tissue, predominantly in the subepicardial layers of the right ventricular (RV) free wall (3). Myocardial biopsy is only performed in a limited number of patients, and, when performed, it is still limited by poor sensitivity (4). Imaging is extensively used in the diagnostic workup of ARVC. To improve the reproducibility of ARVC diagnosis, recent guidelines have introduced quantitative criteria to document RV dilation or ejection fraction (EF) impairment as measured with magnetic resonance (MR) imaging or transthoracic echocardiography (TTE) (2). The presence of intramyocardial fat on multidetector computed tomographic (CT) and MR images has been described for decades (5) but is not part of these recommendations because similar features can also be found in normal hearts (6). To our knowledge, however, tissue characterization with the use of imaging has only been evaluated qualitatively, and a quantitative approach may be more appropriate because the amount of intramyocardial fat is known to be larger in patients with ARVC (7). We hypothesized that the amount of fat in the RV free wall could be quantified with the use of

\section{Advances in Knowledge}

- Automated quantification of right ventricular (RV) fat on multidetector CT images is feasible and accurate for the diagnosis of arrhythmogenic RV cardiomyopathy (ARVC).

- In ARVC, the extent of RV fat is related to RV function $(R=$ $-0.67, P=.001)$, conduction $(R$ $=0.39, P=.02)$ and repolarization $(R=0.38, P=.02)$ disturbances, and PKP2 gene mutation $(R=0.59, P=.02)$. multidetector CT and could be useful as a diagnostic feature in ARVC. The aim of this study was to evaluate an automated method for the quantification of fat in the RV free wall on multidetector CT images and to assess its diagnostic value in ARVC.

\section{Materials and Methods}

\section{Population}

This study was approved by our institutional review board, and all patients gave written informed consent. Between November 2012 and August 2013, we prospectively included 36 consecutive patients referred to our center for the initial diagnosis of ARVC or for the follow-up of a known ARVC (29 men with a mean age \pm standard deviation of 46 years \pm 15 ; seven women with a mean age of 47 years \pm 15$)$. The inclusion criterion was a definite ARVC diagnosis based on current task force criteria (2). Exclusion criteria were contraindications to iodinated contrast media. A second group composed of 36 age- and sex-matched subjects was recruited (seven women aged 47 years \pm 15 ). These patients had been referred for multidetector CT for assessment of the ascending aorta (14 were suspected of having aortic aneurysm, and 22 were undergoing follow-up for a known aneurysm) and had no history of palpitation, syncope, or ventricular ectopy and no history or multidetector CT features that indicated the presence of structural heart disease. A third group composed of 36 patients with ischemic cardiomyopathy (four women aged 62 years \pm 12 ) was recruited (ischemic group, with a diagnosis based on coronary angiography and

\section{Implication for Patient Gare}

- Automated quantification of RV fat from multidetector CT images can be used as a diagnostic feature in ARVC, particularly in patients with implanted cardiac devices when MR imaging may be contraindicated. electrocardiography [ECG] findings). Because the ischemic population differs from the one with ARVC, ischemic and ARVC groups were not matched. In the three groups, all patients underwent two-dimensional (2D) TTE to document left ventricular (LV) EF and $\mathrm{RV}$ fractional area change. In the ARVC group, all patients had undergone signal-averaged ECG and 24-hour Holter monitoring to document ARVC diagnostic criteria. Additionally, 20 of 36 patients had undergone MR imaging, and MR images were reviewed to assess LV EF, RV EF, and LV delayed enhancement. In all groups, LV dysfunction was defined as either LV EF of less than $57 \%$ on MR images (8) or LV EF of less than 55\% at 2D TTE (9). $\mathrm{RV}$ dysfunction was defined as either RV EF of less than $49 \%$ on MR images (10) or RV fractional area change of less than $32 \%$ at 2D TTE (9). Severe $\mathrm{RV}$ dysfunction was defined as RV EF of less than $35 \%$ on MR images, RV fractional area change of less than

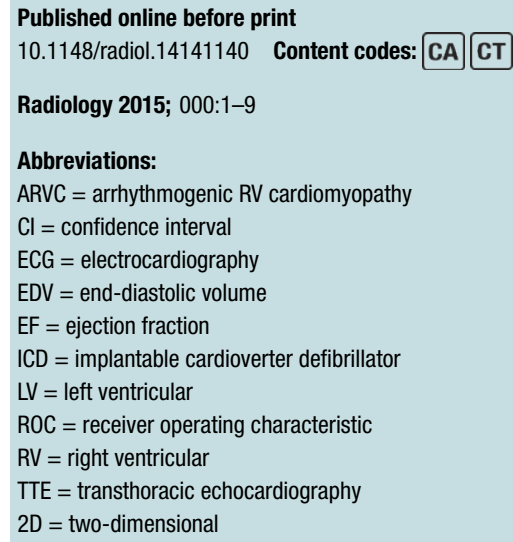

\section{Author contributions:}

Guarantors of integrity of entire study, H.C., A.D., T.A.A., M. Hocini, P.J.; study concepts/study design or data acquisition or data analysis/interpretation, all authors; manuscript drafting or manuscript revision for important intellectual content, all authors; approval of final version of submitted manuscript, all authors; agrees to ensure any questions related to the work are appropriately resolved, all authors; literature research, H.C., Y.K., T.A.A., M. Hocini, F.L.; clinica studies, H.C., A.D., Y.K., A.S.J., T.A.A., F.S., N.D., J.R., O.C. M. Hocini, M. Haïssaguerre, F.L., P.J.; experimental studies, H.C., F.S., M.S.; statistical analysis, H.C., J.R., M.M.; and manuscript editing, H.C., Y.K., F.S., M. Hocini, M. Haïssaguerre, M.M.

Conflicts of interest are listed at the end of this article. 
$18 \%$ at $2 \mathrm{D}$ TTE, or clinical symptoms of RV failure. In patients with ARVC, biventricular dysplasia was defined as either presence of $L V$ dysfunction or evidence of LV fibrosis on delayed contrast material-enhanced MR images.

\section{Multidetector CT Acquisition}

Cardiac multidetector CT was performed by using a contrast-enhanced and retrospectively cardiac-gated method with a 64-section scanner (Somatom Definition; Siemens, Forchheim, Germany). Beta-blockers were used unless contraindicated in every patient who had ventricular ectopics or a heart rate higher than 70 beats per minute. Images were acquired during the first pass of iodinated contrast media (400 mg of iodine per milliliter of iomeprol; Bracco, Milan, Italy). To obtain optimal enhancement of the RV chamber, a biphasic bolus method was applied-that is, 60 $\mathrm{mL}$ of contrast media delivered at the rate of $5 \mathrm{~mL} / \mathrm{sec}$, followed by $80 \mathrm{~mL}$ of a 50:50 mixture of iodinated contrast material and saline at the same rate (11). Typical acquisition parameters were gantry rotation time of $330 \mathrm{msec}$, temporal resolution of $83 \mathrm{msec}$, collimation of $64 \times 0.6 \mathrm{~mm}$, tube voltage $100-120 \mathrm{kV}$, and typical tube current of $600 \mathrm{mAs}$ with a dose modulation protocol. Effective radiation dose was 7.2 $\mathrm{mSv} \pm 4.6,13.2 \mathrm{mSv} \pm 7.1$, and 9.1 $\mathrm{mSv} \pm 5.2$ in the ARVC, control, and ischemic groups, respectively. Images were reconstructed (B26f kernel filter) at end-diastole in a stack of contiguous 1-mm-thick short-axis sections encompassing the whole RV (typical in-plane pixel size, $0.4 \times 0.4 \mathrm{~mm})$.

\section{Quantification of RV Fat}

Image segmentation and fat quantification were performed by using an inhouse method developed as a plug-in of the software OsiriX 3.6.1 (OsiriX Fondation, Geneva, Switzerland). The image-processing strategy is illustrated in Figure 1. After manual definition of tricuspid and pulmonary planes, the RV endocardium was automatically segmented by using region growing. The lower-attenuation threshold applied for region growing was set 3
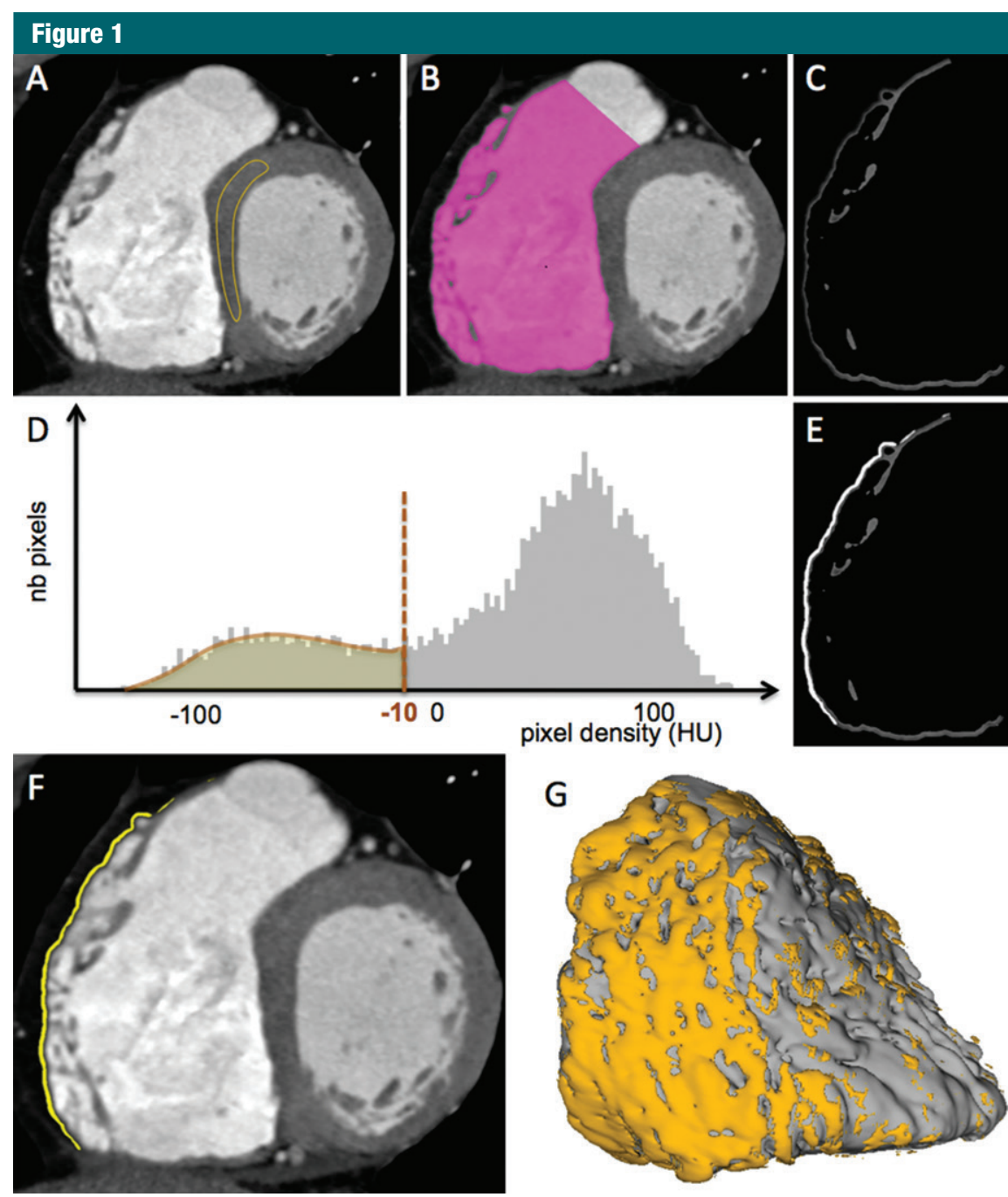

Figure 1: Images demonstrate quantification of intramyocardial fat in the RV free wall in a 36-year-old man. $A$, On short-axis CT images, a $3-\mathrm{cm}^{2}$ region of interest is drawn in a healthy area of the LV myocardium. $B, \mathrm{RV}$ endocardium is then automatically segmented by using region growing, the lower threshold being set 3 standard deviations above mean healthy myocardial attenuation. C, A 2-mm-thick RV free wall layer is automatically segmented by using a dilatation operator. $D, A$ threshold is applied to the histogram of wall pixels to segment $E$, areas exhibiting fat attenuation. $F$, This results in the segmentation of intramyocardial fat over the RV free wall, the extent of fat being expressed as a percentage of the wall. G. Endocardial and fat segmentations are used to compute patient-specific three-dimensional models displaying fat distribution.

standard deviations above mean myocardial attenuation, as assessed by drawing a $3-\mathrm{cm}^{2}$ region of interest in the interventricular septum at midventricular level. A 2-mm-thick RV free wall layer was then automatically derived from RV endocardial segmentation by using a dilatation operator. Myocardial hypoattenuation was automatically segmented in the RV free wall by using histogram thresholding, the cutoff being defined as pixel attenuation of less than $-10 \mathrm{HU}$. This threshold was based on prior validation versus epicardial voltage data in patients with ARVC undergoing electroanatomic mapping with image integration (12). In some patients with implantable cardioverter defibrillators (ICDs), additional manual segmentation was required to remove metal artifacts located at the RV apex. RV 
end-diastolic volume (EDV) was derived from endocardial segmentation and expressed in milliliters per square meter of body surface area. The extent of fat was expressed as percentage of RV free wall. Total processing time per data set was 5 minutes \pm 1 . The reproducibility of fat quantification and the effect of patient size and image noise level on the quantification are analyzed in Appendix E1 (online).

\section{Registration of Fat Distribution on the RV Template}

In all patients, segmentations were used to compute three-dimensional maps that displayed the distribution of myocardial fat over the RV endocardium. To study the distribution of fat in subpopulations, all patient-specific maps were registered to an $\mathrm{RV}$ template based on multidetector CT acquisition in a patient with average RV size and shape. Registration was performed by using landmark-based registration with affine anisotropic transformation, as implemented in Visualization Toolkit, or VTK (Kitware, New York, NY) $(13,14)$. The root mean square error on distance after registration was $3.3 \mathrm{~mm} \pm 3.2$. Registered data sets were then organized according to clinical characteristics to compute statistical maps displaying the likelihood of fat over the $\mathrm{RV}$ wall in ARVC and non-ARVC groups. An illustration of the registration method is provided in the Figure E1 (online). To compare fat likelihood between RV regions, the template was divided into seven segments: apical, mid (anterior, lateral, inferior), and basal (anterior, lateral, inferior).

\section{Statistical Analysis}

The Shapiro-Wilk test of normality and D'Agostino tests for skewness and kurtosis were used to assess whether quantitative data conformed to the normal distribution. Square root transformation was applied in cases of nonnormal distribution. Continuous variables were expressed as means \pm standard deviations. Categorical variables were expressed as fractions (percentages). Continuous variables were compared by using independent-sample parametric (unpaired Student $t$ test) or nonparametric (Mann-Whitney test) tests, depending on data normality. Relationships between variables were assessed by using Pearson or Spearman correlation coefficients $(R)$. Repeated measures analysis of variance and Tukey-Kramer multiple comparison tests were used to compare (a) the global extent of fat between ARVC, ischemic, and control groups; (b) RV EDV between ARVC, ischemic, and control groups; and (c) the extent of fat between segments in each group. The extent of fat between segments in each group was expressed as a percentage of the segment of interest. The diagnostic performance of fat and $\mathrm{RV}$ EDV to diagnose ARVC was assessed by using receiver operating characteristic (ROC) curve analysis. Areas under ROC curves were compared by using $Z$ statistics. All statistical tests were two tailed. A $P$ value less than .05 was considered to indicate a statistically significant difference. Analyses were performed by using NCSS version 8 (NCSS Statistical Software, Kaysville, Utah), except for agreement and correlation ratio analyses $(\mathrm{R}$ statistical software; Foundation for Statistical Computing, Vienna, Austria).

\section{Results}

\section{Population Characteristics}

The characteristics of the ARVC population are provided in Table E1 (online). All patients had a definite ARVC diagnosis based on current task force criteria. Time since first diagnosis was 25 months (range, 0-87 months). Signal-averaged ECG, 24-hour Holter monitoring, and 2D TTE had been performed in all patients within the past month. MR imaging had been performed in 20 patients. The mean time since last MR imaging examination was 12 months (range, 0-42 months) in these patients. Genetic testing for PKP2 mutations had been performed in 16 patients. None of the patients had undergone endomyocardial biopsy. The mean numbers of major and minor criteria at the time of inclusion in the current study were $2.0 \pm 1.3$ and $2.2 \pm 1.2$, respectively.

In the ischemic group (four women with a mean age of 62 years \pm 12 , a mean weight of $82 \mathrm{~kg} \pm 12$, and a body mass index of $28.0 \mathrm{~kg} / \mathrm{m}^{2} \pm 4.3$ ), all patients had a history of prior myocardial infarction. Multivessel disease was present in eight of 36 patients. The right coronary artery was involved in 15 patients, the left anterior descending artery in 18 patients, the circumflex artery in six patients, and the left main artery in three patients. None of the patients exhibited RV dysfunction at TTE. Mean LV EF was $45 \% \pm 22$ at $2 \mathrm{D}$ TTE. The indication for multidetector CT in the ischemic group was the assessment of proximal stents in 16 patients, the detection of intracardiac thrombus in 11 patients, the measurement of aortic aneurysms in six patients, and the characterization of aortic valve stenoses in three patients. In the control group (seven women with a mean age of 47 years \pm 15 , mean weight of $75 \mathrm{~kg} \pm 13$, and body mass index of $24.8 \mathrm{~kg} / \mathrm{m}^{2} \pm 3.5$ ), all patients had been referred for multidetector CT for assessment of the ascending aorta and had no history of palpitation or syncope and no history or multidetector CT features that indicated the presence of structural heart disease. None of the patients exhibited RV dysfunction at TTE. Mean LV EF was $61 \% \pm 7$ at $2 \mathrm{D}$ TTE.

\section{Extent of Fat and RV Volume in ARVC and Non-ARVC}

Multidetector CT measurements of RV fat extent and RV EDV values for each group are presented in Figure 2. RV EDV was $126 \mathrm{~mL} / \mathrm{m}^{2} \pm 38,77 \mathrm{~mL} / \mathrm{m}^{2}$ \pm 19 , and $75 \mathrm{~mL} / \mathrm{m}^{2} \pm 17 \mathrm{~mL} / \mathrm{m}^{2}$ in ARVC, ischemic, and control groups, respectively. RV EDV was higher in the ARVC group versus the ischemic and control groups $(P<.0001$ for both comparisons). No significant difference in RV EDV was found between ischemic and control groups $(P=.61)$. Fat extent was $16.5 \% \pm 6.1,5.0 \% \pm$ 2.7 , and $4.2 \% \pm 2.6$ of $\mathrm{RV}$ free wall in $\mathrm{ARVC}$, ischemic, and control groups, respectively. RV fat extent was higher 


\section{Figure 2}

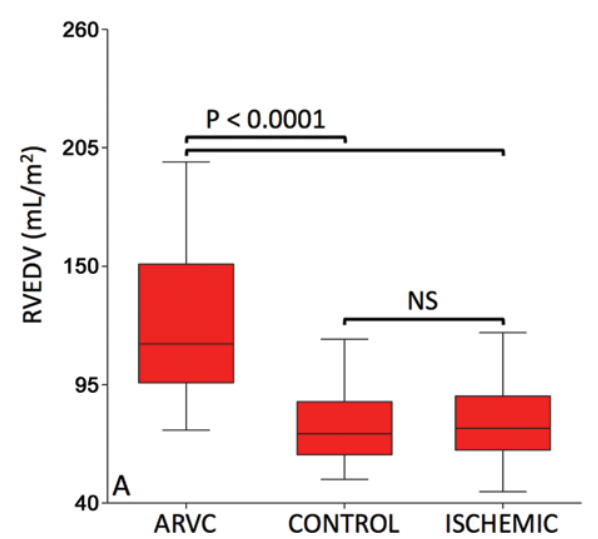

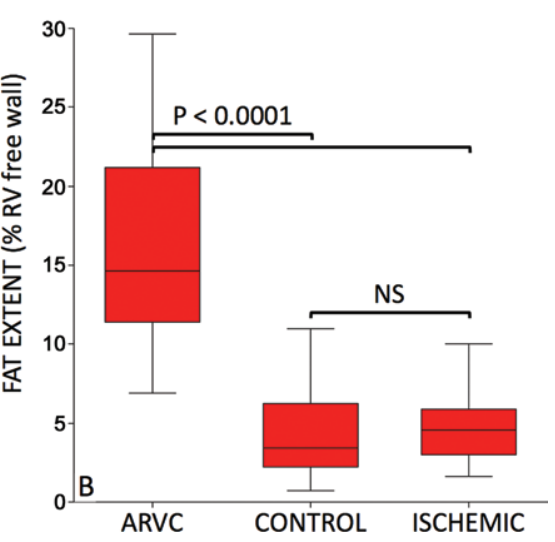

Figure 2: Box and whisker plots show RV EDV and fat extent values in ARVC, control, and ischemic groups. RV EDV was higher in patients with ARVC ( $n=36$; mean age, 46 years \pm 15 ; seven women) versus control subjects ( $n=36$; mean age, 47 years \pm 15 ; seven women) and ischemic patients ( $n=$ 36; mean age, 62 years \pm 12 ; four women) ( $P<.0001$ for both comparisons) and was not significantly different between control subjects and ischemic patients $(P=.61)$. Fat extent in RV free wall was higher in patients with ARVC versus control subjects and ischemic patients $(P<.0001$ for both comparisons) and was not significantly different between control subjects and ischemic patients $(P=.23)$. NS $=$ not significant.

in the ARVC group versus the ischemic and control groups $(P<.0001$ for both comparisons). No significant difference in RV fat extent was found between ischemic and control groups $(P=.23)$. ROC analysis is illustrated in Figure 3. The optimal cutoff value of $8.5 \%$ fat was used to diagnose ARVC with $94 \%$ sensitivity (95\% confidence interval [CI]: $82 \%, 98 \%)$ and $92 \%$ specificity (95\% CI: $83 \%, 96 \%)$. The optimal cutoff value of $106 \mathrm{~mL} / \mathrm{m}^{2} \mathrm{RV}$ EDV was used to diagnose ARVC with 72\% sensitivity (95\% CI: 56\%, 84\%) and $94 \%$ specificity (95\% CI: 87\%, $98 \%)$. The diagnostic performance of fat extent was significantly higher than the one of RV EDV (area under the ROC curves, $0.96 \pm 0.02$ vs $0.88 \pm$ 0.04 , respectively; $P=.009$ ). Of note, fat extent was lower in patients who had a recent diagnosis of ARVC $(<12$ months) than in others $(14.0 \% \pm 4.9$ of RV free wall versus $18.3 \% \pm 6.4$, respectively; $P=.04$ ). As a consequence, the sensitivity in diagnosing ARVC decreased when considering only patients with a recent ARVC diagnosis (sensitivity, 87\%). Figure 4 shows examples of fat quantification in patients with and those without ARVC.

In patients without ARVC, RV fat extent was not found to be related to age $(R=0.10, P=.39), \operatorname{RV} \operatorname{EDV}(R=$ $0.05, P=.65)$, RV systolic function at TTE $(R=-0.04, P=.68)$, LV systolic function at TTE $(R=0.04, P=.69)$, body weight $(R=-0.11, P=.35)$, or body mass index $(R=-0.09, P=.43)$. The correlates of RV fat in ARVC are provided in Table E2 (online). Among diagnostic task force criteria, $\mathrm{RV}$ fat was found to be inversely related to RV EF and positively related to RV EDV, presence of epsilon wave, inverted $\mathrm{T}$ waves in $\mathrm{V}_{1}-\mathrm{V}_{3}$, and $P K P 2$ mutations. RV fat extent was $20.0 \%$ \pm 5.9 when epsilon wave was present and $14.9 \% \pm 5.7$ otherwise $(P=.02)$. It was $18.2 \% \pm 6.3$ when inverted $\mathrm{T}$ waves in $\mathrm{V}_{1}-\mathrm{V}_{3}$ were present and $13.4 \% \pm 4.5$ otherwise $(P=.02)$. It was $20.9 \% \pm 4.3$ when $P K P 2$ mutations were present and $13.8 \% \pm 6.9$ otherwise $(P=.03)$. When analyzing results of multidetector CT-derived fat quantification in patients with ARVC who had also undergone MR imaging ( $n=20$ patients, with positive MR imaging findings in 16), RV fat extent was above the $8.5 \%$ threshold in

\section{Figure 3}

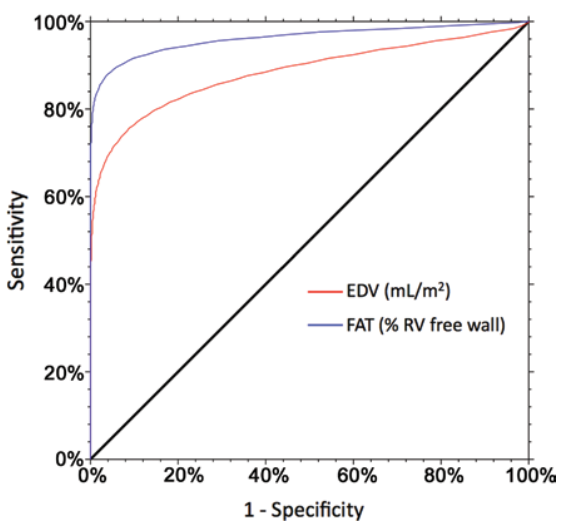

Figure 3: $\quad$ ROC curve is shown for the diagnosis of ARVC. The diagnostic performance of fat extent was higher than that for RV EDV (area under the ROC curves, $0.96 \pm 0.02$ vs $0.88 \pm 0.04$, respectively; $P=.009)$. The optimal cutoff value of $8.5 \%$ fat was used to diagnose ARVC with $94 \%$ sensitivity (95\% Cl: 82\%, 98\%) and $92 \%$ specificity $(95 \% \mathrm{Cl}$ : $83 \%, 96 \%)$. The optimal cutoff value of $106 \mathrm{~mL} /$ $\mathrm{m}^{2}$ RV EDV was used to diagnose ARVC with $72 \%$ sensitivity (95\% Cl: 56\%, 84\%) and 94\% specificity (95\% Cl: 87\%, 98\%).
18 of 20 patients, including two with negative MR imaging findings. The two patients with RV fat extent below the $8.5 \%$ threshold also had negative MR imaging findings.

\section{Distribution of Fat over the RV Free Wall}

The distribution of fat over the RV free wall in patients with ARVC and those without ARVC is illustrated in Figure 5. In patients with ARVC, significant differences were found between RV segments $(F$ ratio $=3.13, P=.008)$. Fat extent was higher in basal lateral segments than in mid lateral and apical segments $(P=.03$ and $P=.001$, respectively), higher in mid anterior segments than in mid lateral and apical segments $(P=.01$ and $P=.0002$, respectively), and higher in basal anterior and basal inferior segments than in apical segments $(P=.04$ and $P=.001$, respectively). In patients without ARVC, significant differences were also found between RV segments $(F$ ratio $=3.89, P=.001)$. Fat extent was higher in mid anterior segments than in basal anterior, basal lateral, basal inferior, and mid inferior segments $(P=$ 


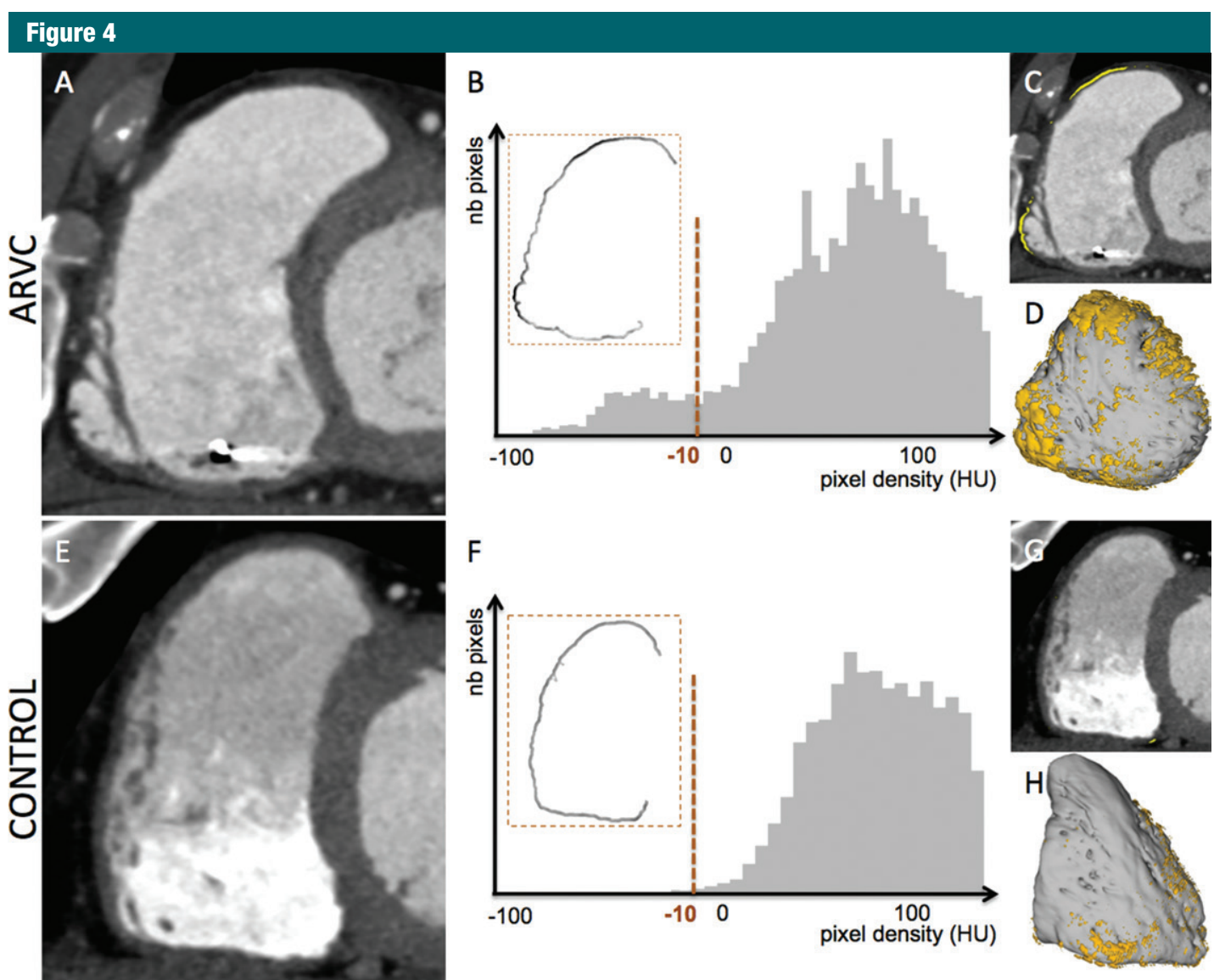

Figure 4: Examples of fat quantification in patients with and those without ARCV are shown. A-D, A 61-year-old man received a diagnosis of ARVC on the basis of task force criteria. $A$, Multidetector short-axis CT image, $B$, histogram analysis, $C$, segmented image, and $D$, patient-specific three-dimensional model in the anteroposterior view are shown. The extent of fat was $12.5 \%$ of RV free wall. RV EDV was $95 \mathrm{~mL} / \mathrm{m}^{2}$. E-H, A 42-year-old man had been referred for cardiac multidetector CT for measurement of the ascending aorta. The patient had no history of palpitation, syncope, or ventricular ectopy and no history or multidetector CT features that indicated the presence of structural heart disease. $E$, Multidetector short-axis CT image, $F$, histogram analysis, $G$, segmented image, and $H$, patient-specific three-dimensional model in the anteroposterior view are shown. The extent of fat was $3.2 \%$ of RV free wall. RV EDV was $71 \mathrm{~mL} / \mathrm{m}^{2}$.

$.005, P=.01, P=.007$, and $P=.02$, respectively). It was also higher in mid lateral and apical segments than in basal anterior segments $(P=.04$ and $P=.04$, respectively).

\section{Discussion}

An automated method for the quantification of intramyocardial fat in the RV free wall from multidetector CT images appears to be feasible. The performance of RV fat extent for the diagnosis of ARVC is superior to that of $\mathrm{RV}$ volume, yielding similar specificity and markedly improved sensitivity. Fat burden is related to RV dysfunction as well as conduction and repolarization disturbances in ARVC.

\section{Diagnosis of ARVC}

Evidence of intramyocardial fat at imaging in ARVC was first reported by Wolf et al in 1989 (15) with the use of MR imaging. Because fat tissue is characterized by low attenuation as compared with the myocardium, intramyocardial fat has also been described on CT images (16). These features were the hallmark of ARVC diagnosis at imaging in the 1990s but were progressively abandoned on the basis of subjectivity and poor reproducibility of the qualitative analysis (17). Additionally, several histologic and imaging reports have described fatty infiltration in patients without ARVC $(5,6)$. For these reasons, the presence of RV fat on images is not part of current recommended criteria to diagnose ARVC (2). In the present study, an automated and quantitative method was used for the assessment of intramyocardial fat in the RV free wall. Although fat can also be assessed at MR imaging, we chose to use multidetector CT because high blood pool attenuation allows for automated endocardial segmentation (18) and because its higher spatial resolution is particularly adapted to the study of the thin RV wall. The choice of a 2-mm thickness in which RV 


\section{Figure 5}
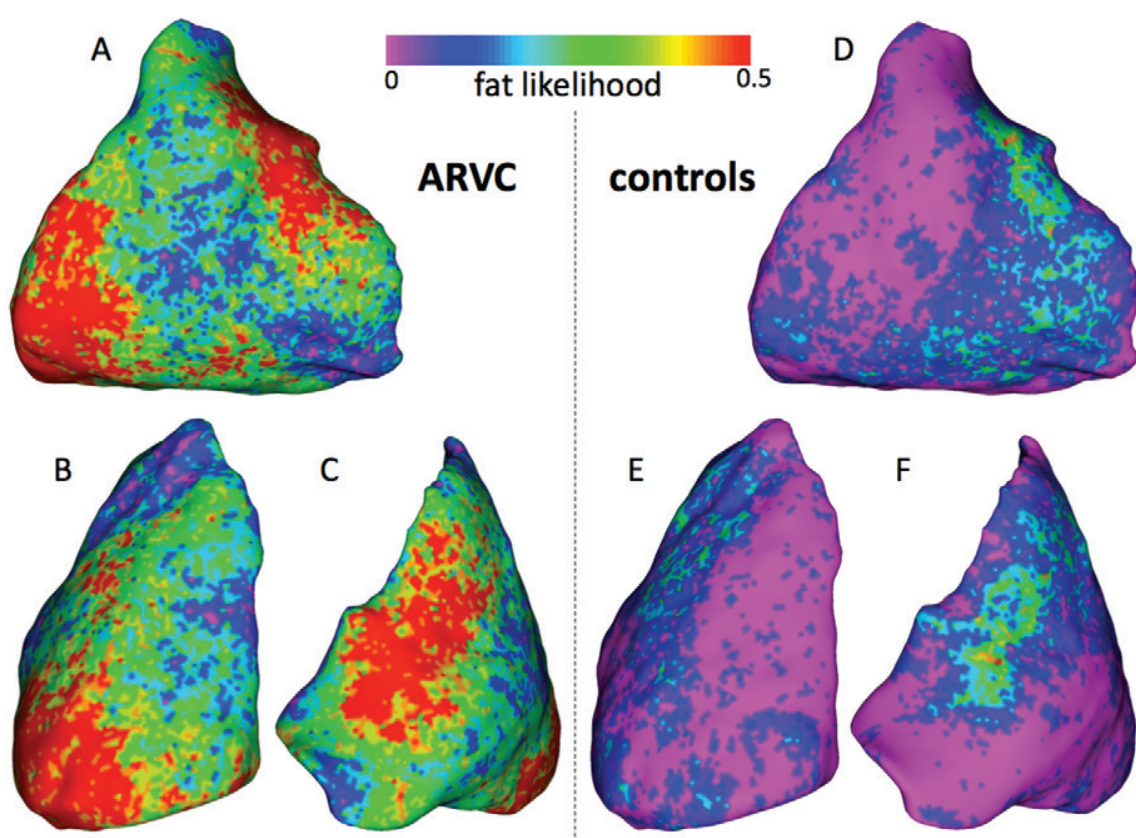

Figure 5: $A-F$, Parametric maps display the distribution of myocardial fat over RV free wall. Fat likelihood is shown for patients with ARVC ( $n=36$; mean age, 46 years \pm 15 ; seven women) and control subjects ( $n$ $=36$; mean age, 47 years \pm 15 ; seven women) in $A, D$, lateral, $B, E$, inferior, and $C, F$, anterior views.

wall densities were analyzed was based on previously reported normal values of RV wall thickness (19). Indeed, because it predominates in subepicardial layers of the myocardium, it is often not possible to discriminate between abnormal intramyocardial fat and normal epicardial fat. Thus, any fat attenuation found less than $2 \mathrm{~mm}$ from the endocardium can be considered abnormal and related to either intramyocardial fat or severe wall thinning. We acknowledge that when analyzing a 2-mm-thick wall, areas of intramyocardial fat located more than $2 \mathrm{~mm}$ from the endocardium are missed. However, the validity of the approach was recently established in a contact mapping study in patients with ARVC who were undergoing ventricular tachycardia ablation, demonstrating high correlation of multidetector CT-derived fat segmentation with voltage mapping and ablation targets (12). The current study demonstrates that the method can also be used for diagnostic purposes, fat quantification being highly reproducible and accurate for the diagnosis of ARVC. The high reproducibility of both $\mathrm{RV}$ EDV and fat measurements can be explained by the limited number of user interactions during the quantification process. The only manual tasks are the definition of a region of interest in a healthy $\mathrm{LV}$ myocardial area to define the lower threshold for subsequent automated blood pool segmentation and the definition of pulmonary and tricuspid planes. Additional studies should be performed to document the reproducibility between different acquisitions in the same patient. For radiation exposure issues, this reproducibility was not explored in the current study.

\section{Clinical Correlates of RV Fat}

When compared with task force criteria, our results indicate that fat quantification performs better than RV EDV in the diagnosis of ARVC, yielding similar specificity (92\% vs 94\%) and markedly improved sensitivity (94\% vs $72 \%)$. The extent of fat was also strongly associated with MR imaging results as expected and yielded positive results in two of four patients with ARVC who had negative MR imaging findings. RV fat extent was also strongly related to the presence of epsilon wave, whereas no relationship was found with late potentials at signal-averaged ECG. This might be explained by the high sensitivity of signal-averaged ECG in the detection of conduction abnormalities even in patients with limited substrate, whereas the presence of epsilon waves at standard ECG might require a larger amount of substrate. The extent of fat was also related to inverted $\mathrm{T}$ waves in $\mathrm{V}_{1}-\mathrm{V}_{3}$, indicating that such repolarization disturbances also require large amounts of substrate to be observed at surface ECG. Interestingly, the extent of fat was found to be higher in patients with PKP2 mutations than in those without. This suggests that the impairment in desmosomal adhesion caused by plakophilin 2 mutations might result in a distinctive phenotype of the disease. Our results show that the extent of fat in the RV free wall is also closely related to RV function, which indicates a potential value for risk stratification (20). However, no relationship was found between the extent of fat in RV free wall and the presence of $L V$ involvement, which is consistent with prior reports of $\mathrm{LV}$ variants of arrhythmogenic cardiomyopathy with minimal RV involvement (21). When analyzing arrhythmic events, the extent of fat in RV free wall was not found to be related to sustained ventricular tachycardia as a first symptom, history of syncope, sudden cardiac arrest, ablation, or ICD. Because only three patients with ICD had not undergone therapy, ICD therapy could not be tested. In prior studies, intramyocardial RV fat was found to be associated with age at histologic examination (22), and myocardial lipid content as measured in the left ventricle with MR imaging spectroscopy was also found to be related to age (23). In the present study, however, no significant correlation was found between RV fat and age, weight, or body mass index. We hypothesized that fat induced by age or metabolic 
disorders is not clustered enough to be quantified by using our multidetector CT approach, because of partial volume effect. Indeed, observing a mean attenuation below $-10 \mathrm{HU}$ in a 0.4 $\mathrm{mm}^{3}$ pixel would require a macroscopically clustered fat infiltrate. Therefore, our method might be adapted to the detection and/or characterization of intramyocardial fat associated with focal diseases (inflammatory, ischemic, dysplasic), but possibly not to a diffuse increase in lipid content secondary to aging or metabolic disorders.

\section{Regional Distribution of Fat over the RV Free Wall}

Our results show that fatty infiltration in ARVC is regionally distributed. Fat predominates on basal lateral and anterior RV outflow tract areas, which is consistent with prior pathologic reports (6). In patients without ARVC, the segmentation also shows a regional distribution of fat densities, with predominance on anterior RV outflow tract. We hypothesize that this is rather due to epicardial fat included in the 2-mm thickness of the wall than to intramyocardial fat. Indeed, anterior RV outflow tract is known to be the area where the RV wall is the thinnest (24). This indicates that when using automated quantification of fat with the proposed method, basal lateral fat is more specific for ARVC than RV outflow tract fat. Therefore, a regional analysis of fat could potentially further improve the performance of the method for diagnosis or disease characterization. Such a regional approach could be of interest to characterize patients with ARVC with different genotypes or different clinical presentations (arrhythmia vs heart failure) or to assist in differential diagnosis (sarcoidosis, RV infarction). These perspectives should be addressed in future studies.

\section{Study Limitations}

The first limitation of this study is related to its case-control design and to the limited sample size. We only studied patients with definite ARVC, task force criteria being used as a reference standard. The value of multidetector $\mathrm{CT}$-derived fat quantification in patients with borderline and possible ARVC diagnosis remains unknown and should be assessed prospectively versus patient outcome. In addition, the time to first diagnosis was variable in the ARVC population, ranging from 0 to 87 months, and it fairly but significantly correlated with fat extent. This reflects the natural disease progression, since initial diagnosis but might have introduced a certain degree of overestimation in sensitivity. Regarding the method, we acknowledge that the proposed segmentation does not quantify septal and LV substrate because it is focused on RV free wall. A quantification of fat in the $L V$ wall would be feasible by using a similar approach, but we believe that qualitative assessment of myocardial fat is sufficient in the left ventricle because it is less limited than on the thin RV wall. Another potential limitation is the absence of functional evaluation at multidetector CT. Indeed, it was shown to be feasible and particularly useful in patients with ICD implants and contraindications to MR imaging (25). A combination with fat quantification would therefore be feasible. However, the radiation dose associated with multidetector CT can be viewed as a limitation, particularly when assessing cardiac motion over the whole cycle. Last, the reproducibility between different acquisitions and different scanners was not specifically analyzed and should be addressed in future studies.

In conclusion, automated quantification of RV fat from multidetector CT images is feasible and could be used to assist the diagnosis of ARVC. In ARVC, fat burden is related to RV function, conduction, and repolarization disturbances and PKP2 mutation.

Disclosures of Conflicts of Interest: H.C. dis closed no relevant relationships. A.D. disclosed no relevant relationships. Y.K. disclosed no relevant relationships. A.S.J. disclosed no relevant relationships. T.A.A. disclosed no relevant relationships. F.S. disclosed no relevant relationships. N.D. disclosed no relevant relationships. J.R. disclosed no relevant relationships. M.S. disclosed no relevant relationships. O.C. disclosed no relevant relationships. M. Hocini. disclosed no relevant relationships. M. Haïssaguerre, disclosed no relevant relationships. F.L. disclosed no relevant relationships. M.M. disclosed no relevant relationships. P.J. disclosed no relevant relationships.

\section{References}

1. Sen-Chowdhry S, Syrris P, Ward D, Asimaki A, Sevdalis E, McKenna WJ. Clinical and genetic characterization of families with arrhythmogenic right ventricular dysplasia/cardiomyopathy provides novel insights into patterns of disease expression. Circulation 2007;115(13):1710-1720.

2. Marcus FI, McKenna WJ, Sherrill D, et al. Diagnosis of arrhythmogenic right ventricular cardiomyopathy/dysplasia: proposed modification of the task force criteria. Circulation 2010;121(13):1533-1541.

3. Marcus FI, Fontaine GH, Guiraudon G, et al. Right ventricular dysplasia: a report of 24 adult cases. Circulation 1982;65(2):384398.

4. Cooper LT, Baughman KL, Feldman AM, et al. The role of endomyocardial biopsy in the management of cardiovascular disease: a scientific statement from the American Heart Association, the American College of Cardiology, and the European Society of Cardiology. J Am Coll Cardiol 2007;50(19): 1914-1931.

5. Kimura F, Matsuo Y, Nakajima T, et al. Myocardial fat at cardiac imaging: how can we differentiate pathologic from physiologic fatty infiltration? RadioGraphics 2010;30(6): 1587-1602.

6. Fontaliran F, Fontaine G, Fillette F, Aouate P, Chomette G, Grosgogeat Y. Nosologic frontiers of arrhythmogenic dysplasia. Quantitative variations of normal adipose tissue of the right heart ventricle [in French]. Arch Mal Coeur Vaiss $1991 ; 84(1): 33-38$.

7. Burke AP, Farb A, Tashko G, Virmani R. Arrhythmogenic right ventricular cardiomyopathy and fatty replacement of the right ventricular myocardium: are they different diseases? Circulation 1998;97(16):15711580 .

8. Maceira AM, Prasad SK, Khan M, Pennell DJ. Normalized left ventricular systolic and diastolic function by steady state free precession cardiovascular magnetic resonance. J Cardiovasc Magn Reson 2006;8(3):417426.

9. Lang RM, Bierig M, Devereux RB, et al. Recommendations for chamber quantification. Eur J Echocardiogr 2006;7(2):79108.

10. Maceira AM, Prasad SK, Khan M, Pennell DJ. Reference right ventricular systolic and diastolic function normalized to age, gender and body surface area from steady-state 
free precession cardiovascular magnetic resonance. Eur Heart J 2006;27(23):28792888.

11. Utsunomiya D, Awai K, Sakamoto T, et al. Cardiac 16-MDCT for anatomic and functional analysis: assessment of a biphasic contrast injection protocol. AJR Am J Roentgenol 2006;187(3):638-644.

12. Komatsu Y, Jadidi A, Sacher F, et al. Relationship between MDCT-imaged myocardial fat and ventricular tachycardia substrate in arrhythmogenic right ventricular cardiomyopathy. J Am Heart Assoc 2014;3(4).

13. Kim EY, Johnson H, Williams N. Affine transformation for landmark based registration initializer in ITK. The MIDAS JournalMedical Imaging and Computing [serial online] http://hdl.handle.net/10380/3299. Published 2011. Accessed January 27, 2012.

14. Horn BK. Closed-form solution of absolute orientation using unit quaternions. $\mathrm{J}$ Opt Soc Am 1987;4(4):629-642.

15. Wolf JE, Rose-Pittet L, Page E, et al. Detection of parietal lesions using magnetic reso- nance imaging in arrhythmogenic dysplasia of the right ventricle [in French]. Arch Mal Coeur Vaiss 1989;82(10):1711-1717.

16. Kimura F, Sakai F, Sakomura Y, et al. Helical CT features of arrhythmogenic right ventricular cardiomyopathy. RadioGraphics 2002;22(5):1111-1124.

17. Bomma C, Rutberg J, Tandri H, et al. Misdiagnosis of arrhythmogenic right ventricular dysplasia/cardiomyopathy. J Cardiovasc Electrophysiol 2004;15(3):300-306.

18. Mühlenbruch G, Das M, Hohl C, et al. Global left ventricular function in cardiac CT. Evaluation of an automated 3D regiongrowing segmentation algorithm. Eur Radiol 2006;16(5):1117-1123.

19. Prakash R. Determination of right ventricular wall thickness in systole and diastole. Echocardiographic and necropsy correlation in 32 patients. Br Heart J 1978;40(11):1257-1261 .

20. Pinamonti B, Dragos AM, Pyxaras SA, et al. Prognostic predictors in arrhythmogenic right ventricular cardiomyopathy: re- sults from a 10-year registry. Eur Heart J 2011;32(9):1105-1113.

21. Sen-Chowdhry S, Syrris P, Prasad SK, et al. Left-dominant arrhythmogenic cardiomyopathy: an under-recognized clinical entity. J Am Coll Cardiol 2008;52(25):21752187.

22. Tansey DK, Aly Z, Sheppard MN. Fat in the right ventricle of the normal heart. Histopathology 2005;46(1):98-104.

23. Sarma S, Carrick-Ranson G, Fujimoto N, et al. Effects of age and aerobic fitness on myocardial lipid content. Circ Cardiovasc Imaging 2013;6(6):1048-1055.

24. Foale R, Nihoyannopoulos P, McKenna W, et al. Echocardiographic measurement of the normal adult right ventricle. Br Heart J 1986; 56(1):33-44.

25. Wu YW, Tadamura E, Kanao S, et al. Structural and functional assessment of arrhythmogenic right ventricular dysplasia/ cardiomyopathy by multi-slice computed tomography: comparison with cardiovascular magnetic resonance. Int J Cardiol 2007;115(3):e118-e121. 